\title{
SQUAMOUS CELL CARCINOMA OF THE BREAST: CASE REPORT
}

Daniel Carbonieri Batista de Oliveira ${ }^{1}$, Carlos Elias Fristachi' ${ }^{1}$ Eveline de Abrantes Silva ${ }^{1}$, Fabio Francisco de Oliveira Rodrigues ${ }^{1}$, Rodrigo Macedo da Silva ${ }^{1}$

IInstituto de Câncer Dr Arnaldo Vieira de Carvalho - São Paulo (SP), Brazil.

Introduction: Primary squamous cell carcinoma of the breast (SCCB) is a rare malignant neoplasm, confirmed when at least $90 \%$ of the cells are of the squamous type. It represents about $1 \%$ of all cases of breast carcinomas. The literature has few reports on the subject, showing that SCCB has no specific radiological features. Objective: To report a case of SCCB diagnosed at the Instituto do Câncer Doutor Arnaldo Vieira de Carvalho. Method: We conducted a review of medical records, photographic records, and a literature review. Result: An 82-year-old woman had a small breast lesion in 2016, but for fear, she hid it until the end of 2018. In the beginning, the lesion was treated with topical corticosteroids and monitored at a community health clinic. After worsening, she was referred to a specialized service. Upon physical examination, the lesion was firm, exophytic, friable, and non-nodular, with fibroelastic axillary lymph nodes of up to 1.5 $\mathrm{cm}$. The mammography from October 2018 was classified as BI-RADS 2 and the breast ultrasound as BI-RADS 3 , showing only skin lesion. Incisional biopsy revealed SCCB with immunohistochemistry positive for cytokeratin-14 and protein p63 and negative for hormone receptors (HR) and HER2 expression. She underwent central quadrantectomy with axillary lymph node dissection in April 2019, confirming SCCB and without lymph node involvement (0/14). After 5 months, the patient showed lesions suggestive of herpes zoster, confirmed by serology. Without improvement with acyclovir, a new biopsy was performed, which confirmed local recurrence. In December, she started radiotherapy, but the lesion progressed to necrosis, papules, and extended to the dorsum, accompanied by intense pain. Chemotherapy with cisplatin $30 \mathrm{mg} / \mathrm{m}^{2}$ weekly was proposed, with partial response. Conclusion: SCCB at diagnosis has $>4 \mathrm{~cm}$, and $50 \%$ of them have associated cysts. Usually, it has a high grade and is negative for HR and HER2 expression. Mammographic images do not have specific features, explaining the large lesions during screening. The literature review revealed that $70 \%$ of SCCB patients do not have lymph node involvement, but due to its unpredictable spread, lymph node dissection might be conducted for staging. It is considered an aggressive disease with an uncertain prognosis, and the data available is not enough to provide options to prevent recurrence. SCCB is rare, has a poor prognosis, and surgery is the most accepted initial treatment. In the case of recurrence, the treatment of choice is radiotherapy associated with cisplatin-based chemotherapy and anti-PD-L1 immunotherapy (cemiplimab), which was recently approved by the Food and Drug Administration (FDA). 